\title{
An Electret Accelerometer for Use in Active Vibration Control Systems
}

\author{
Steven C. Meschia \\ V. Hugo Schmidt \\ Department of Physics \\ Sarah Taubner \\ Department of Electrical Engineering \\ Montana State University \\ Bozeman, MT 59717
}

\begin{abstract}
An electret accelerometer was designed which can easily be integrated with piezoelectric actuators in order to actively control sub-audio vibrations.
\end{abstract}

\section{INTRODUCTION:}

Electrets are employed in nearly ninety percent of commercial microphones. ${ }^{1}$ This technology can be adapted to the manufacture of thin $(<1 \mathrm{~mm}$ thick) transducers which are well suited to the measurement of low-frequency vibrations. Specifically, we are integrating these acceleration sensors with piezoelectric bimorph actuators made from polyvinylidene fluoride.

\section{Motivation}

Commercially available accelerometers tend to be relatively thick (> $1 \mathrm{~mm}$ ), due to their encapsulation of a test mass. We sought to eliminate this excess bulk by using the mass of the object which one seeks to isolate from vibration as a replacement for the encapsulated mass found in most accelerometers. This replacement is valid as long as the controlled mass both lacks excited internal degrees of freedom and remains constant. The first criterion will hold as long as the controlled mass has been effectively isolated from vibrations for a duration long compared to the inverse of the damping parameter. The second criterion holds as long as the controlled mass is encapsulated so that evaporation from the controlled mass cannot occur. Eliminating test masses also allows one to eliminate the rigid containers needed to contain them.

Many commercial accelerometers have unnecessarily high resonant frequencies, reducing their sensitivity at the low frequencies which remain after passive damping of vibration by viscoelastic materials.

The most important criterion which is not met by currently available accelerometers, however, is the prohibitive cost of deploying arrays of accelerometers, which are necessary for correcting for local variations in acceleration across the boundary between the vibration-controlling material and the controlled mass.

0-7803-3355-1/96/\$5.00 @ 1996 IEEE.

\section{DESIGN}

Our accelerometer consists of a circuit board with a highimpedance operational amplifier (Analog Devices AD745) on one side and with two adjacent grounded sections, each measuring $10 \mathrm{~mm}$ by $5 \mathrm{~mm}$, etched onto the other side. On top of these grounded sections is laid an uncharged sheet of $50 \mu \mathrm{m}$ thick FEP (fluorinated ethylene propylene) polymer spacer. On top of this spacer is laid a sheet of $25 \mu \mathrm{m}$ thick FEP polymer which is metalized in two halves only on its bottom surface. Half of this FEP sheet is charged and the adjacent $10 \mathrm{~mm}$ by $5 \mathrm{~mm}$ section remains uncharged. On the corners of the FEP sheet, we placed $50 \mu \mathrm{m}$ tall segments of polyurethane foam, providing an air gap above the charged surface of the FEP film. On top of this is a $50 \mu \mathrm{m}$ thick grounded aluminum plate.

The metalized side of the sheet over the charged portion of the FEP connects to the inverting input of the amplifier and the other metalized half of the sheet connects to the noninverting input of the amplifier. This arrangement provides impedance balance for the amplifier, which maximizes the signal-to-noise ratio of the amplifier. ${ }^{2}$

Both because the only external forces acting on the controlled mass must be transmitted through the accelerometer and because the internal modes of vibration of the controlled mass have small amplitude, the measured voltage will be a function of the movement of the controlled mass. For frequencies well below resonance, the voltage will be proportional to the acceleration of the mass.

\section{THEORETICAL PERFORMANCE}

We assume that the dominant source of noise intrinsic to the sensor system comes from the thermal excitation of electrons in the material of the sensor and the integrated amplifier. The mean square voltage contribution generated by the thermal noise in a frequency interval $\mathrm{df}$ is given by

$$
\mathrm{dV}_{\mathrm{n}}^{2}=4 \mathrm{kT} \operatorname{Re}\{Z\} \mathrm{df},
$$


where $k$ is Boltzmann's constant, $T$ is the temperature, $f$ is the frequency and $Z$ is the impedance of the sensor. If we model the sensor by a parallel RC circuit, then the real part of the impedance at angular frequency $\omega$ equals

$$
\operatorname{Re}\{Z\}=R /\left(1+\omega^{2} R^{2} C^{2}\right) .
$$

Substituting (2) into (1) and assuming an ideal square passband filter with low frequency limit $f_{0}(=0.1 \mathrm{~Hz})$ and highfrequency limit $\mathrm{f}_{1}(=100 \mathrm{~Hz})$, we obtain

$$
\mathrm{V}_{\mathrm{N}}{ }^{2}=(2 \mathrm{kT} / \pi \mathrm{T})\left[\tan ^{-1}\left(2 \pi R C f_{1}\right)-\tan ^{-1}\left(2 \pi R C f_{0}\right)\right] .
$$

For our arrangement, $(\mathrm{RC})_{\mathrm{FEP}}=1.810^{5} \mathrm{~s}$ and $\mathrm{C}=20.7 \mathrm{pF}$, so $\mathrm{V}_{\mathrm{N}}=33 \mathrm{nV}$ at $300 \mathrm{~K}$.

The signal voltage $V_{s}$ is given to a very good approximation by

$$
\begin{gathered}
\mathrm{V}_{s}=\Gamma \sigma \times /\left(\varepsilon_{0} \varepsilon_{1}\right), \\
\Gamma=s_{1}\left(s_{1} s_{2} /\left(\varepsilon_{1}\left(\varepsilon_{2} s_{3}+\varepsilon_{3} s_{2}\right)\right)+s_{2} / \varepsilon_{2} \varepsilon_{3}\right) \\
/\left(s_{1} / \varepsilon_{1}+s_{2} / \varepsilon_{2}+s_{3} / \varepsilon_{3}\right)^{2}
\end{gathered}
$$

where $\Gamma$ is a geometrical factor, $s_{2}$ is the thickness of the charged FEP sheet $(25 \mu \mathrm{m}), s_{3}$ is the air gap $(50 \mu \mathrm{m}), s_{1}$ is the thickness of the FEP spacer $(50 \mu \mathrm{m}), x$ is the acceleration-induced change in the thickness of the air gap, $\sigma$ is the effective surface charge density of the electret, $\varepsilon_{0}$ is the permittivity of the vacuum, $\varepsilon_{1}=\varepsilon_{2}=2.0$ is the relative permittivity of the FEP polymer, and $\varepsilon_{3}=1.0$ is the relative permittivity of air. Because we can vary the modulus of elasticity of the foam-air gap by varying the fraction of foam which covers the surface, we determined the displacement by setting the resonant frequency $f_{\text {es }}$ at twice the highest frequency which we wish to control: $f_{\text {es }}=2 f_{1}=200 \mathrm{~Hz}$. For our underdamped accelerometer system, the acceleration for $f$ $\leq \mathrm{f}_{\mathrm{f}}$ is approximately $4 \pi^{2} \mathrm{f}^{2} \mathrm{x}$. We obtain a theoretical signal voltage of $41 \mu \mathrm{V}$ for an acceleration of $1 \times 10^{-5} \mathrm{~m} \mathrm{~s}^{-2}$.

The intrinsic signal-noise-ratio SNR, defined as $10 \mathrm{log}$ $\left(V_{s}^{2} / V_{n}^{2}\right)$, is thus $61.7 \mathrm{~dB}$ for a $1 \times 10^{-5} \mathrm{~m} \mathrm{~s}^{-2}(\cong 1 \mu \mathrm{g})$ acceleration at any frequency high enough so that appreciable charge does not leak off the FEP electrode through the amplifier input resistance $R_{a}\left(R_{a}=3 \times 10^{11} \Omega, R C=11 \mathrm{~s}\right)$, we assume a surface charge density value of $10^{-3} \mathrm{C} \mathrm{m}^{-2}$, which has been reported to be sustainable in FEP. ${ }^{3}$

If we include the noise of the integrated amplifier $\mathrm{V}_{\mathrm{Na}}\left(\mathrm{V}_{\mathrm{Na}}=\right.$ $0.44 \mu \mathrm{V}$ referred to the amplifier input), we obtain a theoretical performance level of $39.4 \mathrm{~dB}$ for $1 \times 10^{-5} \mathrm{~m} \mathrm{~s}^{-2}$ acceleration.

\section{MEASURED PERFORMANCE}

In order to determine the noise level, we assumed that the charged FEP polymer did not produce significantly more noise than the uncharged polymer. The polymer was discharged by submerging it in warm soap water and then dried without wiping the surface. This film was placed inside the sensor and the signal appearing on the oscilloscope was attributed to the thermal noise. This method may overestimate the noise level because of the difficulty of avoiding all triboelectric charging of FEP film. With an internal amplifier gain of 100 , an RMS noise voltage of 0.1 $\mathrm{mV}$ was obtained in this way. This yielded a noise level of $1 \mu \mathrm{V}$ over the entire $10 \mathrm{kHz}$ frequency band of the sensor. This result agrees well with the calculated noise value of the accelerometer $\left.\left(\left(\mathrm{V}_{\mathrm{N}}{ }^{2}+\mathrm{V}_{\mathrm{Na}}\right)^{2}\right)^{1 / 2}\right)$ over a measured bandwidth of $10 \mathrm{kHz}: 0.9 \mu \mathrm{V}$.

The film was then charged in a $5 \mathrm{MeV} \mathrm{m}^{-1}$ field at $100 \propto \mathrm{C}$. The film was replaced in the sensor and placed on a electromechanical shaker. Instead of foam inserts supporting the top ground plate, the top plate was suspended above the charged FEP polymer. This eliminated the complexity of the mechanical resonance of the sensor. A Kistler $5 \mathrm{~g}$ Piezotron accelerometer was used for calibrating the acceleration of the shaker. The voltage output was then recorded as a function of frequency. A large gap was necessary in order to avoid contacting the front surface of the electrode during the vibration tests. The air gap $s_{3}$ was approximately one millimeter, with variations of as much as $50 \mu \mathrm{m}$ resulting from wrinkles in the FEP, which were produced during the bonding of the FEP to the bottom electrodes. This geometry gives a theoretical value $\mathrm{dV}_{\mathrm{s}} / \mathrm{ds}_{3}$ of $3.4510^{4} \mathrm{~V} / \mathrm{m}$ for a $\sigma$ of $10^{-3} \mathrm{C} / \mathrm{m}^{2}$. The peak voltage $V_{s}$ was read from the oscilloscope at a frequency high enough so that charge leakage was negligible. The peak variation in $s_{3}$ is acc/ $\omega^{2}$, where acc is the peak acceleration and $\omega$ is the angular frequency of the shaker.

Our measurements indicated a value of $\mathrm{dV}_{\mathrm{S}} / \mathrm{ds}_{3} 3.5$ times higher than given above, suggesting a $\sigma$ of $3.510^{-3} \mathrm{C} / \mathrm{m}^{2}$. This value is higher than the quoted ${ }^{3}$ sustainable charge density of $10^{-3} \mathrm{C} / \mathrm{m}^{2}$. It was obtained on a freshly charged sample and decayed considerably within the following days. With our current charging apparatus, we are unable to adequately stabilize the charge on the FEP sheet by heating the material to the glass transition $\left(190^{\circ} \mathrm{C}\right)$ because, well below the glass transition, the tensile strength of the polymer decreases to the point where it become impossible to pull the FEP sheet through the electrodes. 


\section{CURRENT WORK}

We are experimenting with new ways of charging the film, namely corona charging using the electron beam from a JEOL Model JSM6100 Scanning Microscope, and are measuring the charge densities obtained.

\section{REFERENCES}

[1] P. Edwards, AT\&T press release, <http://www.attcom /press/1295 1951228.blahtml>, December 1995.

[2] AD745 Application Note, Analog Devices, One Technology Way, P.O Box 9106, Norwood, MA, p.10, 1995.

[3] G. M. Sessler and J. E. West., "Production of high quasipermanent charge densities on polymer foils by application of breakdown fields," $J$. Appl. Phys., vol. 43, p. 922, March 1972. 
